\title{
Producing cluster states in charge qubits and flux qubits
}

\author{
Tetsufumi Tanamoto, ${ }^{1}$ Yu-xi Liu, ${ }^{2}$ Shinobu Fujita, ${ }^{1}$ Xuedong $\mathrm{Hu},{ }^{3}$ and Franco Nori ${ }^{2,4}$ \\ ${ }^{1}$ Corporate $R$ \& $D$ center, Toshiba Corporation, Saiwai-ku, Kawasaki 212-8582, Japan \\ ${ }^{2}$ Frontier Research System, The Institute of Physical and Chemical Research (RIKEN), Wako-shi, Saitama 351-0198, Japan \\ ${ }^{3}$ Department of Physics, University at Buffalo, SUNY, Buffalo, New York 14260-1500,USA \\ ${ }^{4}$ Physics Department, MCTP, The University of Michigan, Ann Arbor, Michigan 48109-1040, USA
}

(Dated: June 26, 2018)

\begin{abstract}
We propose a method to efficiently generate cluster states in charge qubits, both semiconducting and superconducting, as well as flux qubits. We show that highly-entangled cluster states can be realized by a 'one-touch' entanglement operation by tuning gate bias voltages for charge qubits. We also investigate the robustness of these cluster states for non-uniform qubits, which are unavoidable in solid-state systems. We find that quantum computation based on cluster states is a promising approach for solid-state qubits.
\end{abstract}

PACS numbers: 03.67.Lx, 03.67.Mn, 73.21.La

One-way quantum computing [1], which is based on a series of one-qubit measurements starting from cluster states of a qubit array, is an intriguing alternative to the widely studied approach using unitary quantum gates. Here, the power of quantum mechanics, such as quantum parallelism and entanglement, is already stored in the initial cluster state. Cluster states are fixed highlyentangled states that involve all qubits and act as a universal resource for quantum computing.

Because of their unique importance, cluster states have been studied in a variety of physical systems. They have been extensively explored in optical quantum computers both theoretically [2] and experimentally [3]. By incorporating cluster states, optical quantum computing can achieve substantially simpler operations [2] compared to the original linear optics quantum computing proposal 4]. Cluster states have also been studied in solid state qubits. In particular, processes of generating cluster states for single and encoded spin qubits have been proposed [5, 6] using the Heisenberg exchange interaction.

The existing methods of generating cluster states all require multiple steps because of the types of interaction involved (in the case of photonic qubits, a large number of optical elements is also required). Here we describe theoretically an efficient method to create scalable cluster states in charge qubits $7,8,9,10,11,12,13$ ] and flux qubits [14, 15, 16], using existing Ising-like interactions. Our key result is that cluster states in charge qubits can be created by applying a single gate bias pulse, right after preparing an initial product state $\left|\Psi_{0}\right\rangle \equiv|\Psi(t=0)\rangle=$ $\Pi_{i}|+\rangle_{i}$, where $| \pm\rangle_{i}=\left(|0\rangle_{i} \pm|1\rangle_{i}\right) / \sqrt{2}$. We also calculate the time-dependent fidelity of the cluster states in charge qubits using a quantum dot (QD) system with decoherence produced by the measurement back-action, and explore the effects of non-uniformity among qubits, which is a realistic characteristics for all solid-state qubits.

Cluster states in charge qubits. - The Hamiltonian for an array of charge qubits with nearest-neighbor interac- tions is described by

$$
H_{\mathrm{cq}}=\sum_{i}\left(\Omega_{i} \sigma_{i x}+\epsilon_{i} \sigma_{i z}\right)+\sum_{i<j} J_{i j} \sigma_{i z} \sigma_{j z}
$$

with Pauli matrices $\sigma_{i x}$ and $\sigma_{i z}$ for the $i$-th qubit. $\Omega_{i}$ is either the inter-QD tunnel coupling for coupled QD systems 7, 8, 9], or half the Josephson energy for superconducting charge qubits 11, 12, 13], respectively. For either semiconducting or superconducting charge qubits, $\epsilon_{i}$ is the charging energy, and corresponds to the energy difference between $|0\rangle$ and $|1\rangle$ for each qubit. The coupling constants $J_{i j}$ are derived from the capacitance couplings. In one-way quantum computing [1], the $\sigma_{i x}$ term needs to be switched off during the creation of the cluster state $\left(H_{\mathrm{cs}}\right)$, and then switched on when measurements are carried out. From this perspective, charge qubits with tunable $\Omega_{i}$ [17] are desirable. However, tunability can produce decoherence and cross-talk between qubits themselves and between qubits and the environment. In addition, for most qubit systems, once the qubit array is made, $\Omega_{i}$ and $J_{i j}$ are fixed, and only $\epsilon_{i}$ is controllable via the gate voltage bias (we call these "simple-design qubits"). Practically, such a simple design is preferable for solid-state qubits so as to simplify fabrication and enhance scalability. Thus, our goal here is to generate cluster states for simple-design qubits. Hereafter, without loss of generality, and for convenience, we focus on qubits of coupled QDs. $|0\rangle$ and $|1\rangle$ refer to the two singlequbit states in which the excess charge is localized in the upper and lower dot, respectively (see Ref. [9]).

Typically, cluster states are generated by an Ising-like Hamiltonian $H_{\mathrm{cs}}=(g / 4) \sum_{i<j}\left(1-\sigma_{i z}\right)\left(1-\sigma_{j z}\right)$, where $i$ and $j$ are nearest-neighbor sites, starting from an initial state $\left|\Psi_{0}\right\rangle$. Preparing a unitary evolution $U_{\mathrm{cs}}(t)=$ $\exp \left(i t H_{\mathrm{cs}}\right)$ (we use $\left.\hbar=1\right)$ at $g t=\left(2 n_{\mathrm{I}}+1\right) \pi$, where $n_{\mathrm{I}}$ is an integer, is the first step for one-way quantum computing. Since simple-design charge qubits have Ising-like interactions, all we need to do to get $H_{\mathrm{cs}}$ out of Eq. (11) is to turn off the effect of the $\sigma_{x}$ terms in (1). This can 
be achieved in the high-bias regime where $\epsilon_{i} \gg \Omega_{i}>J_{i j}$, by applying a canonical transformation to Eq. (11) [18]:

$$
H_{\mathrm{cq}}^{(\mathrm{eff})} \approx H_{\mathrm{cq}}+\left[S, H_{\mathrm{cq}}\right]=\sum_{i} E_{i} \sigma_{i z}+\sum_{i<j} J_{i j} \sigma_{i z} \sigma_{j z}+H_{\mathrm{uw}}
$$

where $S=-i \sum_{i}\left[\Omega_{i} /\left(2 \epsilon_{i}\right)\right] \sigma_{i y}$, with $\Omega /\left(2 \epsilon_{i}\right) \ll 1$. Also, $E_{i}=\epsilon_{i}+\Omega_{i}^{2} / \epsilon_{i}$, neglecting terms of higher-order than $\left(\Omega_{i} / \epsilon_{i}\right)^{2} . \quad H_{\mathrm{uw}}$ is an unwanted interaction term given by $H_{\mathrm{uw}}=$ $-\sum_{i=1}^{N} \sum_{\vec{\gamma}_{d}}\left(\Omega_{i} / \epsilon_{i}\right) J_{i, i+\vec{\gamma}_{d}} \sigma_{i x} \sigma_{i+\vec{\gamma}_{d}, z}$ for a $d$-dimensional qubit array. Here $\vec{\gamma}_{1}= \pm 1, \vec{\gamma}_{2}=\{( \pm 1,0),(0, \pm 1)\}$ and $\vec{\gamma}_{3}=\{( \pm 1,0,0),(0, \pm 1,0),(0,0, \pm 1)\}$ are nearest neighbor indices for one-, two- and three-dimensional qubit arrays, respectively. As long as $H_{\mathrm{uw}}$ is sufficiently small and can be neglected, we can periodically generate cluster states in the tilted frame $|\tilde{\Psi}(t)\rangle=e^{-S}|\Psi(t)\rangle$ after a time $t_{\mathrm{cs}}$, if both $J t_{\mathrm{cs}}=\pi / 4+2 n_{\mathrm{J}} \pi$ and $E_{i} t_{\mathrm{cs}}=-(\pi / 4) \bar{n}_{i}+2 n_{\mathrm{E}} \pi$ are satisfied $\left(\bar{n}_{i}\right.$ is the number of qubits connected to the $i$-th qubit; $n_{\mathrm{J}}(\geq 0)$ and $n_{\mathrm{E}}$ are arbitrary integers, and $J_{i j}$ should be uniform: $\left.J_{i j}=J\right)$. These two equalities lead to the relation $J\left(8 n_{\mathrm{E}}-\bar{n}_{i}\right) /\left(8 n_{\mathrm{J}}+1\right)=E_{i} \equiv E_{i, t_{\mathrm{cs}}}$. Thus, to generate a cluster state, gate bias voltage $\epsilon_{i}$ for the $i$-th qubit needs to be set at

$$
\epsilon_{i}=\epsilon_{i}^{\mathrm{cs}}=\frac{E_{i, t_{\mathrm{cs}}}}{2}+\sqrt{\left(\frac{E_{i, t_{\mathrm{cs}}}}{2}\right)^{2}-\Omega_{i}^{2}}
$$

during $t_{\mathrm{cs}}=\pi\left(8 n_{\mathrm{J}}+1\right) /(4 J)$. To ensure a solution for $\epsilon_{i}^{\mathrm{cs}}$ exists, we require $n_{\mathrm{E}}-\left(2 \Omega_{i} / J\right) n_{\mathrm{J}}>\left(2 \Omega_{i} / J+\bar{n}_{i}\right) / 8$. Hereafter we choose to treat the case of $n_{\mathrm{J}}=0$, which corresponds to the shortest possible time to generate cluster states. To further ensure the high-bias regime, where $\epsilon_{i} \gg \Omega_{i}$, we require $8 n_{\mathrm{E}}-\bar{n}_{i} \gg 2 \Omega_{i} / J$.

An initial product state $|\tilde{\Psi}(0)\rangle=\left|\Psi_{0}\right\rangle$ has to be prepared for qubits other than the input qubits [1]. $|\Psi(0)\rangle=$ $\Pi_{i}\left(\cos \left[\left(\Omega_{i} /\left(2 \epsilon_{i}\right)+\pi / 4\right]|0\rangle+\sin \left[\left(\Omega_{i} /\left(2 \epsilon_{i}\right)+\pi / 4\right]|1\rangle\right)\right.\right.$ is the corresponding state in the original $\{|0\rangle,|1\rangle\}$ basis, which can also be adjusted by the gate bias on each qubit.

For example, to obtain the two-qubit cluster state $|\Psi\rangle_{C_{2}}=\left(|0\rangle_{1}|+\rangle_{2}+|1\rangle_{1}|-\rangle_{2}\right) / \sqrt{2}$, where $\bar{n}_{1}=\bar{n}_{2}=1$, we can use Eq. (3) and choose a gate bias $\left(\epsilon_{1}^{\mathrm{cs}}=\epsilon_{2}^{\mathrm{cs}}\right)$ from $\{6.37 J, 14.7 J, \ldots\}\left(n_{\mathrm{E}} \geq 1\right)$ for $\Omega=2 J$, and $\{13.8 J, 22.3 J, \ldots\}\left(n_{\mathrm{E}} \geq 2\right)$ for $\Omega=4 J$, etc.

Our approach is valid as long as the unwanted term $H_{\text {uw }}$ can be neglected. We can estimate a lifetime, beyond which we lose the cluster state due to the presence of $H_{\mathrm{uw}}$, by calculating the fidelity defined by $F(t)=\left\langle\Psi_{0}\left|e^{i H_{\mathrm{cs}} t} e^{-i\left(H_{\mathrm{cs}}+H_{\mathrm{uw}}\right) t}\right| \Psi_{0}\right\rangle \approx 1-$ $i t\left\langle\Psi_{0}\left|H_{\mathrm{uw}}\right| \Psi_{0}\right\rangle+(1 / 2)(i t)^{2}\left\langle\Psi_{0}\left|\left[H_{\mathrm{uw}}, H_{\mathrm{cs}}\right]+H_{\mathrm{uw}}^{2}\right| \Psi_{0}\right\rangle$. For a $d$-dimensional $N$-qubit array,

$$
F(t) \approx 1-\left(\frac{\Omega}{\epsilon} J t\right)^{2} 4 d N
$$

Thus, the lifetime of the cluster state is limited by $t<$ $t_{\mathrm{uw}} \equiv(2 J(\Omega / \epsilon) \sqrt{d N})^{-1}$. Furthermore, the constraint $t_{\mathrm{cs}}<t_{\mathrm{uw}}$ imposes a limit on the number of clustered qubits: $N_{\max }<(2 \epsilon /(\pi \Omega))^{2} / d$. For example, consider a one-dimensional qubit-chain with $\Omega_{i}=4 J$, and $\bar{n}_{i}=2$. For $n_{\mathrm{E}}=2\left(\epsilon_{i}^{\mathrm{cs}} / J \approx 12.7\right), N_{\max }=4$. For $n_{\mathrm{E}}=6$ $\left(\epsilon_{i}^{\mathrm{cs}} / J \approx 45.6\right), N_{\max }=52$. Indeed, we can choose an infinite number of bias conditions for each set of fixed $\Omega_{i}$ and $J$. These are closely related to the possible number $N_{\max }$ of clustered qubits and to the scalability of the system. Various kinds of errors, as discussed in Ref. [2], should be taken into account for more detailed estimates.

As shown in Ref. [9], $J_{i j}$ and $\Omega_{i}$ are determined by the distances between QDs. $J_{i j}$ is directly determined by the capacitance network of QDs and basically controlled as a linear function of the distances between QDs. $\Omega_{i}$ depends exponentially on the distances between two QDs in a qubit. With current technology it is quite difficult to fabricate an array of QDs with very uniform $\Omega_{i}$. For superconducting charge qubits the situation is similar. However, notice that our approach does not require $\Omega_{i}$ to be extremely uniform since we can adjust $\epsilon_{i}$ according to $\Omega_{i}$ in order to obtain an appropriate $E_{i}$ in Eq. (2). In addition, as noted above, $n_{\mathrm{E}}$ can be selected arbitrarily, which adds flexibility to our scheme. Thus, the one-touch cluster state generation method we discuss here should work with any charge qubit architecture. In short, although in general charge qubits have shorter decoherence times compared with spin qubits, our simpler and faster generation method could make them competitive with spin qubits in the context of cluster states, since several steps are required to generate cluster states for spin qubits [5, 6].

Measurement scheme in charge qubits.- In one-way quantum computing, calculations are carried out by a series of local measurements in the $\sigma_{x}$ and $\sigma_{y}$ eigenbasis. For most charge qubits, however, the measurement is carried out in the $\sigma_{z}$ eigenbasis $\{|0\rangle,|1\rangle\}$ by simply applying a large gate bias and using field-effect detectors such as quantum point contacts (QPCs) or single-electron transistors. Thus, for charge qubits the $\sigma_{x}$ and $\sigma_{y}$ basis measurements should be converted into $\sigma_{z}$ measurements after rotating the frame via $\pm(\pi / 2)_{y}$ and $\pm(\pi / 2)_{x}$ pulses. These pulses can be generated by applying AC gate biases such as $\epsilon_{i}(t)=\epsilon_{0 i} \cos \left(\omega_{i c} t+\phi_{i}\right)$. In a rotating coordinate $U_{\mathrm{rw}}(t)=\exp \left(-i \sum_{i} \omega_{i c} t \sigma_{i x}\right)$, the wave function is given by $|\tilde{\Psi}(t)\rangle=U_{\mathrm{rw}}^{\dagger}|\Psi(t)\rangle$, and the Hamiltonian on resonance $\left(\omega_{i c}=\Omega_{i}\right)$ is given by:

$$
H_{\mathrm{rw}} \approx \sum_{i} \frac{\epsilon_{i 0}}{2}\left(\sigma_{i z} \cos \phi_{i}-\sigma_{i y} \sin \phi_{i}\right)+H_{y z},
$$

where $H_{y z}=\sum_{i j}\left(J_{i j} / 2\right)\left(\sigma_{i y} \sigma_{j y}+\sigma_{i z} \sigma_{j z}\right)$ is an unwanted term here. In order to realize $\left|\Psi\left(t_{\mathrm{m}}\right)\right\rangle=e^{ \pm i \frac{\pi}{4} \sum_{i} \sigma_{i y}}|\Psi(0)\rangle$ at the measurement time $t=t_{\mathrm{m}}$, we should have $e^{i H_{\mathrm{rw}} t_{\mathrm{m}}} \propto e^{i \sum_{i} \Omega_{i} t_{\mathrm{m}} \sigma_{i x}} e^{\mp i \frac{\pi}{4} \sum_{i} \sigma_{i y}}$. Thus, we obtain $t_{\mathrm{m}}=$ $(\pi / \Omega) l_{1}$, the voltage amplitude $\epsilon_{i 0}=\left(\pi / 2 t_{\mathrm{m}}\right)\left(1+4 l_{2}\right)$, and the phase $\phi_{i}=\pi / 2+l_{3} \pi\left(l_{1}, l_{2}\right.$ and $l_{3}$ are arbitrary 
integers $\left.\left(l_{1} \neq 0\right)\right)$ for the $\sigma_{x}$ measurement. If we take $t_{\mathrm{m}}=(\pi /(4 \Omega))\left(1+4 l_{1}\right)$ and $\epsilon_{i 0}=2 \pi l_{2} / t_{\mathrm{m}}\left(l_{2} \neq 0\right)$, we realize the $\sigma_{y}$ measurement. By applying a gate bias after the time $t_{\mathrm{m}}, \sigma_{x}$ and $\sigma_{y}$ measurements are processed. This scheme works well for the region where $\epsilon_{i 0} / J \gg 1$.

For the experiment in Ref. [13], $\Omega=20.5 \mu \mathrm{eV}$ and $J=$ $95 \mu \mathrm{eV}$, and thus $t_{\mathrm{cs}}=\pi /(4 J)=34.2 \mathrm{psec}, \epsilon_{\mathrm{cs}} \sim 664 \mu \mathrm{eV}$ from Eq. (3) at $n_{\mathrm{E}}=1$ and $t_{\mathrm{uw}}=(\pi / J)\left(\epsilon_{\mathrm{cs}} /[2 \sqrt{2} \Omega]\right)=$ $1.57 \mathrm{nsec}$ for a dephasing time of the order of $T_{2} \sim 5$ nsec. Thus, our approach can be applied, if smaller $J$ $(<\Omega)$ is prepared, for instance, by increasing the distance between qubits.

Cluster states in flux qubits. - For flux qubits, $\Omega>\epsilon$ in Eq. (1), so that here we cannot directly use the abovementioned one-touch approach for charge qubits. Here we show a method to generate cluster states for flux qubits by applying an oscillating magnetic field. Consider two inductance-coupled flux qubits working at the optimal bias [16] with Hamiltonian:

$$
\begin{aligned}
H_{\mathrm{fq}} & =\epsilon_{1} \sigma_{1 z}+\epsilon_{2} \sigma_{2 z}+\Omega_{1}^{R} \cos \left(\omega_{1}^{\mathrm{rf}} t+\phi_{1}\right) \sigma_{1 x} \\
& +\Omega_{2}^{R} \cos \left(\omega_{2}^{\mathrm{rf}} t+\phi_{2}\right) \sigma_{2 x}+J_{x x} \sigma_{1 x} \sigma_{2 x}
\end{aligned}
$$

where $\Omega_{i}^{R}$ and $\omega_{i}^{\text {rf }}$ are the half amplitude and the frequency respectively of the applied classical field. At the optimal bias point, the system is immune, up to first order, to variations on the control parameters and is thus robust against decoherence. This Hamiltonian is a good starting point for generating cluster states. In the rotating wave approximation for two identical qubits $\left(\Omega_{1}=\Omega_{2}\right)$, we have $\tilde{H}_{\mathrm{fq}}=H_{0}+H_{x y}$, with $H_{0}=\sum_{i=1}^{2}\left(\Omega_{i}^{R} / 2\right)\left(\sigma_{i x} \cos \phi_{i}+\sigma_{i y} \sin \phi_{i}\right)$ and $H_{x y}=$ $J_{x x}\left(\sigma_{1 x} \sigma_{2 x}+\sigma_{1 y} \sigma_{2 y}\right)$. The operator to generate cluster states, $U_{\mathrm{cs}}$, is produced by switching on and off the resonant field of $\Omega_{i}^{R}$ and controlling the phase $\phi_{i}$ similarly to the conventional conditional phase gate operation. For example, if we define $R_{i \alpha}(\theta) \equiv \exp \left(i \theta \sigma_{i \alpha}\right),(\alpha=x, y)$, and $U_{x y}(\theta) \equiv \exp \left(i \theta\left(\sigma_{1 x} \sigma_{2 x}+\sigma_{1 y} \sigma_{2 y}\right)\right)$, we have:

$$
\begin{aligned}
\tilde{U}_{\mathrm{cs}} & =R_{1 x}\left(\theta_{1}\right) R_{2 x}\left(\theta_{2}\right) U_{x y}\left(\theta_{3}\right) R_{1 x}\left(\theta_{4}\right) U_{x y}\left(\theta_{5}\right) R_{1 x}\left(\theta_{6}\right) \\
& =\exp \left(-i \pi\left(\sigma_{1 x}+\sigma_{2 x}-\sigma_{1 x} \sigma_{2 x}\right) / 4\right)
\end{aligned}
$$

with $\theta_{1}=\theta_{2}=-\pi / 4, \theta_{3}=\theta_{5}=\pi / 8, \theta_{4}=\pi / 2$ and $\theta_{6}=-\pi / 2$. After rotating $\tilde{U}_{\mathrm{cs}}$ around the $y$-axis, we recover the original cluster state generator $U_{\mathrm{cs}}$. In the case of many qubits, cluster states for the entire system are generated by applying Eq. (7) to all the neighboring qubit pairs. Note that Eq. (6) also describes the rotating wave approximation to the charge qubits in Eq. (15). Thus, this method of generating cluster states is also applicable to charge qubits, and not only to flux qubits.

The time required for the creation of a cluster state in flux qubits is $T_{\text {flux }}=5 \pi /\left(2 \Omega_{1}^{R}\right)+\pi /\left(4 \Omega_{2}^{R}\right)+\pi /\left(4 J_{x x}\right)$. Taking $\Omega_{1}^{R} \sim \Omega_{2}^{R} \sim J_{x x} \sim 0.5 \mathrm{GHz}$, we obtain $T_{\text {flux }} \sim 18$ nsec $\left(T_{2} \sim 200\right.$ nsec 15$\left.]\right)$. The effect of imperfect pulses can be estimated by substituting $\theta_{j} \rightarrow \theta_{j}+\delta_{j}$ in Eq. (77).

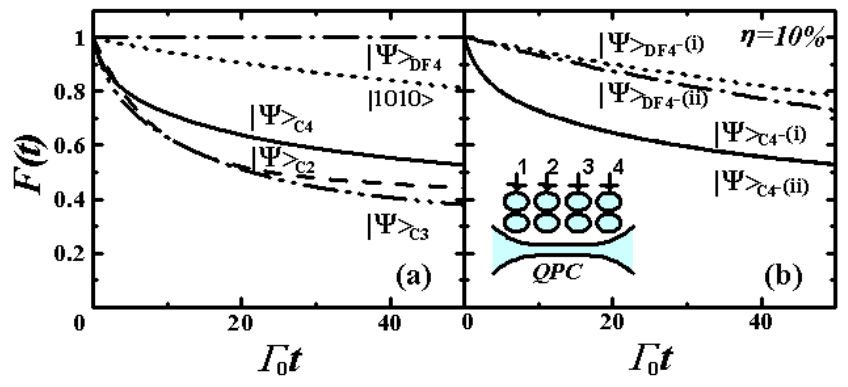

FIG. 1: Time-dependent fidelities $F(t)$ of cluster states $|\Psi\rangle_{\mathrm{C} 2},|\Psi\rangle_{\mathrm{C} 3},|\Psi\rangle_{\mathrm{C} 4}$, a product state $|1010\rangle$ and four-qubit decoherence-free $(\mathrm{DF})$ states $|\Psi\rangle_{\mathrm{DF} 4}=(|1100\rangle-|1001\rangle-$ $|0110\rangle+|0011\rangle) / 2$, for $\Gamma_{0}=J$ and $\Delta \Gamma=0.6 J . \quad \Omega=4 J$ and $n_{\mathrm{E}}=2$ in Eq. (3), thus $\epsilon_{2}=\epsilon_{3} \sim 12.7 \mathrm{~J}, \epsilon_{1}=\epsilon_{4} \sim 13.8 \mathrm{~J}$. (a) Comparison of the cluster states, DF state and $|1010\rangle$. (b) The case when non-uniformity in the qubit parameters is introduced as $\Omega_{i}=4 J\left(1-\eta_{i}\right), \epsilon_{i}=\epsilon+\eta_{i} J$ and $\Gamma_{i}^{( \pm)}=\left(1-\eta_{i}\right) \Gamma^{( \pm)}$, with $i$ indicating the $i$-th qubit. Here $\eta_{i}=0$ for all qubits besides $\eta_{3}=0.1$ (i) and $\eta_{4}=0.1$ (ii). The fidelities of $|\Psi\rangle_{\mathrm{C} 4}$ for (i) and (ii) mostly overlap. (inset) Four qubits that use double dot charged states are capacitively coupled to a QPC detector. We consider a similar detection setup for two and three qubits. These calculations are carried out by the $H_{\mathrm{cq}}$, that is, they include $H_{\mathrm{uw}}$ and higher order terms.

If we take the deviation from a perfect pulse as $\delta_{1}=\delta_{2}$, $\delta_{3}=\delta_{5}, \delta_{4}=\delta_{6}$, for the initial state $\left|\Psi_{0}\right\rangle$, the fidelity is given by

$$
F(t) \approx 1-\delta_{1}^{2} / 2-2 \delta_{3}^{2}+\sin ^{2}[\pi / 8]\left(\delta_{4}^{2} / 2\right)+i \delta_{1} \delta_{4} e^{i \pi / 8}
$$

Thus, the fidelity $F(t)$ remains close to one, up to second order in the pulse shape error, even when the pulse shape has defects.

Effect of non-uniformity in cluster states.- Cluster states are highly entangled states involving all qubits, and can be decohered by various kinds of local fluctuations. Here we investigate the effect of non-uniform qubit parameters on cluster states in semiconductor QDs, from Eq. (1), using a measurement setup, which produces decoherence in the double dot qubits through backaction. We analyze a capacitively coupled detector (such as a QPC), whose shot-noise constitutes a random charge fluctuation on the qubits.

We use a density matrix (DM) to describe up to four qubits (inset of Fig 10 19]. The DM equations for the qubits and the QPC detector are derived in Ref. [19]:

$$
\begin{aligned}
\frac{d \rho_{z_{1}, z_{2}}}{d t} & =i\left[K_{z_{2}}-K_{z_{1}}\right] \rho_{z_{1}, z_{2}}-i \sum_{j=1}^{N} \Omega_{j}\left(\rho_{g_{j}\left(z_{1}\right), z_{2}}-\rho_{z_{1}, g_{j}\left(z_{2}\right)}\right) \\
& -\left[\Gamma_{z_{1}}^{1 / 2}-\Gamma_{z_{2}}^{1 / 2}\right]^{2} \rho_{z_{1}, z_{2}}
\end{aligned}
$$

where $z_{1}, z_{2}=(1111),(1110), \ldots,(0000)$ for four qubits (256 equations) and $z_{1}, z_{2}=(11),(10),(01),(00)$ for two 
qubits (16 equations). $K_{z_{1}}$ is the energy of the $z_{1}$ state and depends on $\epsilon_{i}$ and $J_{i j}$ in Eq. (11). For example, for two qubits, $K_{(11)}=\epsilon_{1}+\epsilon_{2}+J_{12}$ while $K_{(10)}=\epsilon_{1}-\epsilon_{2}-J_{12}$. The $g_{j}\left(z_{i}\right) \mathrm{s}$ are introduced for notational convenience and are determined by the relative positions between qubit states. We assume that the tunneling rate $\Gamma$ of the QPC detector in the presence of $N$ qubits satisfies $\Gamma^{-1}=\sum_{i} \Gamma_{i}^{-1}$, where the tunneling rate $\Gamma_{i}$ is determined by the state $\sigma_{i z}= \pm 1$ of the $i$-th qubit. The strength of the measurement can be parametrized by $\Delta \Gamma_{i}$ as $\Gamma_{i}^{( \pm)}=\Gamma_{i 0} \pm \Delta \Gamma_{i}$, where $\Gamma_{i 0}$ is the tunneling rate of the QPC in the absence of the qubits. The time-dependent fidelity $F(t) \equiv \operatorname{Tr}[\hat{\rho}(0) \hat{\rho}(t)]$ can be calculated by tracing over the elements of the reduced DM obtained from Eq. (9). $F(t)$ can be expanded in time as $F(t)=1-\sum_{n=1}(1 / n !)\left(t / \tau^{(n)}\right)^{n}$, where the lifetime is $1 / \tau^{(n)}=\left\{-\operatorname{Tr}\left[\hat{\rho}(0) d^{n} \hat{\rho}(0) / d t^{n}\right]\right\}^{1 / n}$.

From Eq. (9), we obtain the first-order lifetime for two-, three- and four-qubit cluster states $|\Psi\rangle_{C_{2}}$, $|\Psi\rangle_{C_{3}}=\left(|+\rangle_{1}|0\rangle_{2}|+\rangle_{3}+|-\rangle_{1}|1\rangle_{2}|-\rangle_{3}\right) / \sqrt{2}$, and $|\Psi\rangle_{C_{4}}=$ $\left(|+\rangle_{1}|0\rangle_{2}|+\rangle_{3}|0\rangle_{4}+|+\rangle_{1}|0\rangle_{2}|-\rangle_{3}|1\rangle_{4}+|-\rangle_{1}|1\rangle_{2}|-\rangle_{3}|0\rangle_{4}+\right.$ $\left.|-\rangle_{1}|1\rangle_{2}|+\rangle_{3}|1\rangle_{4}\right) / 2$, respectively, as follows:

$$
\begin{aligned}
1 / \tau_{C 2}^{(1)} & =\sum_{z_{1}, z_{2}=(11), . .,(00)} \Gamma_{\mathrm{d}}\left(z_{1}, z_{2}\right) / 8 \\
1 / \tau_{C 3}^{(1)} & =\sum_{z_{1}, z_{2}=(111), \ldots,(000)} \Gamma_{\mathrm{d}}\left(z_{1}, z_{2}\right) / 32 \\
1 / \tau_{C 4}^{(1)} & =\sum_{z_{1}, z_{2}=(1111), \ldots,(0000)} \Gamma_{\mathrm{d}}\left(z_{1}, z_{2}\right) / 128
\end{aligned}
$$

where the dephasing rate is defined as $\Gamma_{\mathrm{d}}\left(z_{1}, z_{2}\right) \equiv\left[\Gamma_{z_{1}}^{1 / 2}-\right.$ $\left.\Gamma_{z_{2}}^{1 / 2}\right]^{2}$. Note that the lifetime of the cluster states is an average over all the dephasing rates between different product states. This is in contrast with other entangled states. For example, the lifetime of two-qubit Bell states $|c\rangle=(|10\rangle+|01\rangle) / \sqrt{2}$ and $|d\rangle=(|10\rangle-|01\rangle) / \sqrt{2}$ takes the form $1 / \tau_{c}^{(1)}=1 / \tau_{d}^{(1)}=(1 / 2) \Gamma_{\mathrm{d}}(10,01)$. It is well known that the singlet state $|d\rangle$ is the most robust two-qubit state [20] when there is a symmetry between qubits. However, solid-state qubits generally decohere due to various kinds of local causes, which often break the symmetry of the qubit state. Our results in Eq. (1012) indicate that cluster states might be robust against non-uniformity or local defects because of the averages.

In Fig. 1 we compare the fidelity of cluster states with a product state and a four-qubit decoherence-free (DF) state 20]. Figure 1(a) shows the time-dependent fidelities of two-qubit and four-qubit cluster states and the product state $|1010\rangle$, when $\Gamma_{0}=J$ and $\Delta \Gamma=0.6 J$. Our results show that the strongly entangled cluster states are more fragile than a product state such as $|1010\rangle$. We can also see that the robustness of the cluster state depends on the number of qubits in the cluster state. Figure 1(b) shows the time-dependent fidelities of both cluster states and DF states, for non-uniform qubits. Here the parameters $\Omega_{i}, \epsilon_{i}$ and $\Gamma_{i}$ for the third or fourth qubit deviate from those of other qubits by $10 \%$. Note that the fidelities of $|\Psi\rangle_{\mathrm{C} 4}$ show almost the same behavior irrespective of the distribution of the non-uniformity. Furthermore, a comparison between Fig. M(a) and प(b) shows that the non-uniformity has almost no effect on the fidelity of the $|\Psi\rangle_{\mathrm{C} 4}$ cluster state. These results vividly illustrate our analytical analysis of the lifetime (that it is an average over all the product states). In contrast, in Ref. 19] we showed that the robustness of the DF states strongly depends on the non-uniformity. Thus, even though cluster states are generally more fragile than DF states, they are more robust against non-uniformities among qubits than DF states.

In conclusion, we describe how to efficiently generate cluster states in solid-state qubits. By manipulating the gate bias voltage, we explicitly show how to generate 'one-touch' entanglement via cluster states in charge qubits. We also investigate the robustness of cluster states, and find that one-way quantum computing could be viable for solid-state qubits.

FN and XH are supported in part by NSA, LPS, ARO, and NSF.

[1] H. J. Briegel and R. Raussendorf, Phys. Rev. Lett. 86, 910, 5188 (2001); R. Raussendorf, D. E. Browne, and H. J. Briegel, Phys. Rev. A 68, 022312 (2003).

[2] M.A. Nielsen, Phys. Rev. Lett. 93, 040503 (2004); C. M. Dawson et al., ibid, 96, 020501 (2006); M. A. Nielsen and C. M. Dawson, Phys. Rev. A 71, 042323 (2005); M. S. Tame et al., ibid, 72, 012319 (2005); Y. Tokunaga et al., ibid, 71, 030301(R) (2005).

[3] P. Walther et al., Nature (London) 434, 169 (2005).

[4] E. Knill et al., Nature (London) 409, 46 (2001).

[5] M. Borhani and D. Loss, Phys. Rev. A 71, 034308 (2005).

[6] Y. S. Weinstein, C. S. Hellberg, and J. Levy, Phys. Rev. A 72, 020304 (2005).

[7] J. Gorman et al., Phys. Rev. Lett. 95, 090502 (2005).

[8] T. Hayashi et al., Phys. Rev. Lett. 91, 226804 (2003).

[9] T. Tanamoto, Phys. Rev. A 64, 062306 (2001).

[10] B. Koiller et al., Phys. Rev. B 73, 045319 (2006).

[11] Y. Nakamura et al., Phys. Rev. Lett. 88, 047901 (2002).

[12] Y. Makhlin et al., Rev. Mod. Phys. 93, 357 (2001).

[13] T. Yamamoto et al., Nature (London) 425, 941 (2003).

[14] A. Izmalkov et al., Phys. Rev. Lett. 93, 037003 (2004); J. B. Majer et al., ibid, 94, 090501 (2005).

[15] Y. X. Liu et al., Phys. Rev. Lett 96, 067003 (2006).

[16] C. Rigetti et al., Phys. Rev. Lett. 94, 240502 (2005).

[17] J. Q. You and F. Nori, Phys. Today 58 (11), 42 (2005).

[18] We take the first order of the expansion $e^{-S} H_{\mathrm{cq}} e^{S}=$ $H_{\mathrm{cq}}+\left[S, H_{\mathrm{cq}}\right]+\left[S,\left[S, H_{\mathrm{cq}}\right]\right] /(2 !) \ldots$

[19] T. Tanamoto and S. Fujita, Phys. Rev. B 72, 085335 (2005).

[20] P. Zanardi and M. Rasetti, Phys. Rev. Lett. 79, 3306 (1997); D. A. Lidar et al., ibid 82, 4556 (1999). 